\title{
El uso de las redes sociales está cada vez más extendido en la educación en salud y en la práctica clínica
}

Social media use increasingly grows in health education and clinical practice

\section{Introducción}

Durante los últimos años, las redes sociales han empezado a ocupar un lugar importante como estrategia para el acceso a la información y han cambiado la forma en la que se crea la información y se comparte entre los usuarios.

Este estudio es una revisión tipo mapeo (Scoping Review) e intenta evaluar la información sobre la extensión y la naturaleza de la evidencia disponible y discutir la necesidad de nuevas perspectivas de abordaje.

\section{Objetivos de esta revisión}

Mapear la literatura existente respecto al uso de redes sociales por los profesionales de la salud. Determinar la extensión y tipo de evidencia disponible e identificar las necesidades de futuras investigaciones.

\section{Métodos}

Un investigador se ocupó de revisar 12 bases de datos incluyendo a MEDLINE, CENTRAL, ERIC y PubMed, sin tener restricción por

Tabla 1: Características de los estudios incluidos en cuanto al uso de las redes sociales por profesionales de la salud.

\begin{tabular}{l|c|c}
\multicolumn{1}{c|}{ Caracteristica } & Todos los estudios & Excluyendo foros de discusión \\
\hline Promedio de fecha de comienzo & 2006 & 2008 \\
\hline Duración del estudio & 4 meses & 3 meses \\
\hline Diseño del estudio & Cuantitativo: ECCA (5) & Cuantitativo: ECCA (1) \\
(número de estudios) & Corte transversal (41) & Corte transversal (28) \\
& Cualitativo (42) & Favoreció la comunicación (30) \\
& Favoreció la comunicación (62) & Neutral (16) \\
Conclusiones de los autores & Neutral (24) & Empeoró la comunicación (4) \\
& Empeoró la comunicación (6) & Indeterminado (3) \\
\hline
\end{tabular}

En cuanto al propósito del uso de las redes sociales, se observó que en un $61,5 \%$ de los estudios el objetivo fue la comunicación entre pares, instructor-estudiante y entre médicos y pacientes. Cabe destacar que el $92,6 \%$ de los estudios en Blogs o microblogs y el $93,6 \%$ de los estudios en redes sociales se focalizaron principalmente en la comunicación.

En 41 estudios se evaluó el conocimiento y en 19 estudios la mejoría en habilidades. idioma o tipo de estudio encontrado. Se hizo búsqueda de estudios publicados entre 2000 y 2012. Se encontraron 14.365 estudios de los cuales se rastrearon títulos y resúmenes para evaluar la elegibilidad y se excluyeron 12.445 estudios. Se clasificaron el texto como: Relevante, No relevante o No claro.

En el caso de existir alguna discrepancia, se resolvía por consenso de los investigadores. Finalmente quedaron incluidos 96 estudios que tenían algún tipo de investigación primaria.

\section{Resultados}

En este estudio se dividió a las redes sociales en: proyectos cooperativos (Wikipedia); blogs (Twitter); comunidades de contenidos (YouTube); social networking (Facebook) y juegos virtuales o mundos sociales (Second Life).

También se incluyeron a los foros de discusión pero no entraron dentro de esta clasificación.

La información se sintetizó en forma descriptiva y de acuerdo a los distintos grupos (ver tabla 1).

\section{Conclusión}

El uso de las redes sociales está cada vez más extendido en la educación en salud y en la práctica clínica. El principal objetivo de su uso es para facilitar la comunicación.

Sin embargo, las herramientas más populares (Facebook y Twitter) no son las más utilizadas para este propósito y pocos estudios evalúan la confidencialidad y la privacidad de las informaciones.

Fuente de financiamiento: Knowledge Synthesis Grant from the Canadian Institutes of Health Research.

\section{Comentario}

El impacto creciente de uso de redes sociales sobre las actividades de los profesionales de la salud ha motivado que las instituciones sanitarias y los profesionales investiguen la utilidad y contenido de estos recursos de la era digital.

Esta revisión plantea un punto de partida para futuras investigaciones acerca del uso de las redes sociales y otras herramientas de web por los profesionales de la salud y ofrece una primera visión de la información disponible y abre las puertas para el futuro.

Hay que destacar que en estas futuras investigaciones las implicancias éticas y de privacidad deberían ser evaluadas ya que muchas veces, son esos temas lo que dificultan el avance de la utilización de estas valiosas herramientas en la práctica clínica y en la educación en salud.

Por otra parte no puede faltar el desarrollo de estudios de eficacia que nos permita obtener resultados duros de la utilización de estos recursos.

Finalmente hay que tener en cuenta que es muy difícil seguir el ritmo de los avances de las distintas herramientas disponible dado el vertiginoso progreso de las redes sociales.

Ruíz Yanzi María Victoria [ Médica de Familia del Servicio de Medicina Familiar y Comunitaria del Hospital Italiano de Buenos Aires mariav.ruiz@hospitalitaliano.org.ar ]

Ruiz Yanzi M. El uso de las redes sociales está cada vez más extendido en la educación en salud y en la práctica clínica. Evid Act Práct Ambul. 2014, 17(2). Abr-Jun. 60. Comentado de: Hamm MP, Chisholm A, Shulhan J, y col. Social media use by health care professionals and trainees: a scoping review. Acad Med. 2013 Sep;88(9):1376-83. doi: 10.1097/ACM.0b013e31829eb91c. PMID: 23887004. 